\title{
KONGRESSKALENDER
}

\section{August 2021}

\section{3.-27.08.2021, Virtuelle Konferenz}

European Association of Psychology and Law (EAPL)

30th Annual Conference

Information: http://eapl.eu

25.-27.08.2021, Frankfurt am Main,

Goethe-Universität

Hybridveranstaltung, auch virtuell

16th Conference International Association

for the Treatment of Sexual Offenders

(IATSO)

Prof. Dr. Martin Rettenberger

Information: www.iatso.org/index.php/

frankfurt-2021

30.08.-01.09.2021, Zürich, Schweiz

ISFPP - 12. Internationales Symposium

Forensische Psychiatrie

World Trade Center Zürich

PD Dr. A. Rossegger, Prof. Dr. J. Endrass, A.

Nägeli, Prof. Dr. M. Graf

Information: www.forensiktagung.ch

\section{September 2021}

02.-04.09.2021, Hannover

17. Fachtagung der Kriminologischen Gesellschaft (KG)

Kriminalität und Digitalisierung -

Digitalisierung und Kriminalität

Tagungszentrum Schloss Herrenhausen

Prof. Dr. Bliesener

www.kfn.de/KrimG21
08.-11.09.2021, Bukarest, Rumänien European Association of Criminology 19th Annual ESC Conference (II)legal organizations and crime Information: http://www.esc-eurocrim.org/

\subsubsection{1, Berlin-Mitte}

24. Berliner Junitagung für Forensische Psychiatrie und Psychologie

Die erheblich verminderte Schuldfähigkeit §21 StGB -

Wie lässt sie sich erfahrungswissenschaftlich begründen?

Forensische Psychiatrie Psychologie Berlin e.V. (FPPB) \&

Institut für Forensische Psychiatrie der

Charité

Langenbeck-Virchow-Haus, aber auch

virtuell

Information: www.forensik-berlin.de

(Veranstaltungen)

29.09.-02.10.2021, USA

Association for the Treatment of Sexual Abusers (ATSA)

40th Annual Research and Treatment Conference

Information: http://www.atsa.com/futureatsa-conferences

\section{Oktober 2021}

08.10.2021, München

36. Herbsttagung für Forensische

Psychiatrie

Abteilung für Forensische Psychiatrie der

Universität München

Prof. Dr. K. Schiltz

Information: www.forensik-muenchen.de
21.10.2021, Dresden

25. Dresdner Frühjahrstagung für

Forensische Psychiatrie

Theorie und Praxis der forensischen Psychopathologie -

Zum 100. Geburtstag von Werner Janzarik Prof. Dr. Saß, Dr. Lammel, Dr. Lange, Prof. Dr. Bauer, Dr. Sutarski

Information: www.forensik-dresden.de

\section{1.-23.10.2021, Virtuelle Konferenz}

American Academy of Psychiatry and the Law

52nd Annual Meeting (with APA)

Information: www.AAPL.org

\section{November 2021}

05./06.11.2021, Lüneburg

Jenseits von Beziehung? Dissozialiät in psychiatrischen Kontexten

Norddeutschen Arbeitsgemeinschaft Psychodynamische Psychiatrie (NAPP) Ort: Lüneburg, Psychiatrisches Krankenhaus Information: Holger Selig <holgselig@ aol.com>

17.-20.11.2021 Chicago (USA)

American Society of Criminology - 76th

Annual Meeting

Science and Evidenced-Based Policy in a Fractured Era

Chicago, Palmer House Hilton

Information: https://asc41.com/events/2021asc-annual-meeting/

Hinweise für den Kongresskalender bitte an: mail@hlkroeber.de 